\title{
Internet y la necesidad de una nueva teoría psicolingüística
}

Luis Barrera Linares'

lbarrera@usb.ve

\section{RESUMEN}

Este artículo propone una discusión sobre la necesidad de reformulación de la teoría psicolingüística y literaria actual y de los comunes ejes conceptuales en que se.han fundamentado las propuestas estructuralistas, generativistas y discursivas (cognición, identidad, actos de habla, participantes, relaciones lógicas, direccionalidad, linealidad, tiempo, espacio) frente a los eventos propios de la comunicación virtual. Luego de la emergencia e implantación de la comunicación a través de la red, parece imperativa una minuciosa revisión y actualización de los postulados de la psicolingüística general. Nociones como las de lengua, variedad, literatura, género y narración parecen requerir ya de nuevos parámetros operativos que den cuenta de su aparición en el ciberespacio y de sus implicaciones dentro de las Ilamadas comunidades virtuales. Una nueva modalidad de hablantes-lectores virtuales se está gestando a través de la red y está incidiendo a su vez en la modificación de las variables en que se sustentó la teoría lingüística del siglo xx.

\section{Palabras clave}

Teoría psicolingüística, Internet, comunicación, comunidad virtual, recreación literaria.

\section{ABSTRACT}

This paper proposes a discussion about the necessity of reformulating contemporary psycholinguistic and literary theory and the common conceptual axes that structuralist, generative and discursive proposals have relied upon (cognition, identity, speech acts, context, speakers, authors, logic relationships of directionality, linearity, time and space), regarding the events that characterize virtual communication. A new kind of virtual speaker-reader is emerging in the Internet and modifying some concepts on which $20^{\text {TH }}$ century theoretical linguistics stood.

\section{KEYWORDS}

Linguistic theory, Internet, communication, virtual community, literary re-creation

1 Universidad Simón Bolívar-Academia Venezolana de la Lengua. Especialista en Investigación Lingüística y Literaria. Magíster en Psicolingüística. Dr. en Letras. Profesor titular. 


\section{Introducción}

T loda teoría acerca de determinado fenómeno, proceso o estado de cosas intenta de alguna manera explicar lo que a entender de la disciplina que la sustenta debe significar la realidad. Esta premisa es válida desde alguna concepción particular de la filosofía más abstracta hasta la que intente dar cuenta de la existencia de algún objeto concreto, palpable, mensurable y cuantificable.

A su vez. ello implica la necesidad de que las teorías preexistentes se debiliten, caduquen, se modifiquen o evolucionen de acuerdo con los cambios de ordenamiento de la misma realidad que intentan explicar o de nuevos paradigmas científicos que obliguen a revisitarlas $y$, de ser procedente, a reformularlas y adecuarlas, sustituirlas o desecharlas. $Y$ esto simplemente porque como bien ha expresado David Bohm (1980), la realidad no es propiamente un escenario fijo e inmodificable, sino más bien un estado de cosas cuya sustancia es cambiante.

Podríamos recordar que la aparición en la historia de la cultura de nuevas formas tecnológicas como el libro, el telégrafo, la máquina de escribir, el teléfono, el fax, la radio y la televisión modificaron los modos clásicos y más convencionales de lo que hasta esos momentos se definía como (sistemas de) comunicación oral o escrita. Esto naturalmente obligó a los teóricos de la información y la comunicación social a abrir espacios científicos que dieran cabida a tales nuevas formas. No por azar, se comenzó a hacer patente la noción de la aldea global o de la "galaxia $\mathrm{Gu}^{-}$ tenberg” de la que alguna vez habló $\mathrm{M}$. McLuhan (1969), aparte de que la propia ciencia de la información sufrió los reajustes a que daba lugar el cambio de las relaciones entre emisores de mensajes y receptores.

La finalidad principal de este artículo se relaciona con el hecho de que, si bien ya hemos aceptado que el surgimiento de Internet ha significado (o significará en muy breve lapso) el fenómeno tecnológico de mayor impacto para la cultura humana, superando incluso aquellos cambios inimaginables que se dieron, primero, con la aparición de la escritura, y, después, con la invención de la imprenta y el libro impreso, todavía, a tres lustros de la oferta pública de la World Wide Web (Castells, 2000; Mounier, 2002, y Barrera Linares, 2005), la lingüística y la literatura se están alimentando de las mismas bases teóricas que las sustentaron durante casi todo el siglo xx: desde la irrupción del estructuralismo saussureano hasta las más recientes orientaciones del análisis del discurso, pasando por la teoría generativo-transformacional.

\section{El lenguaje está cambiando, mas no la teoría}

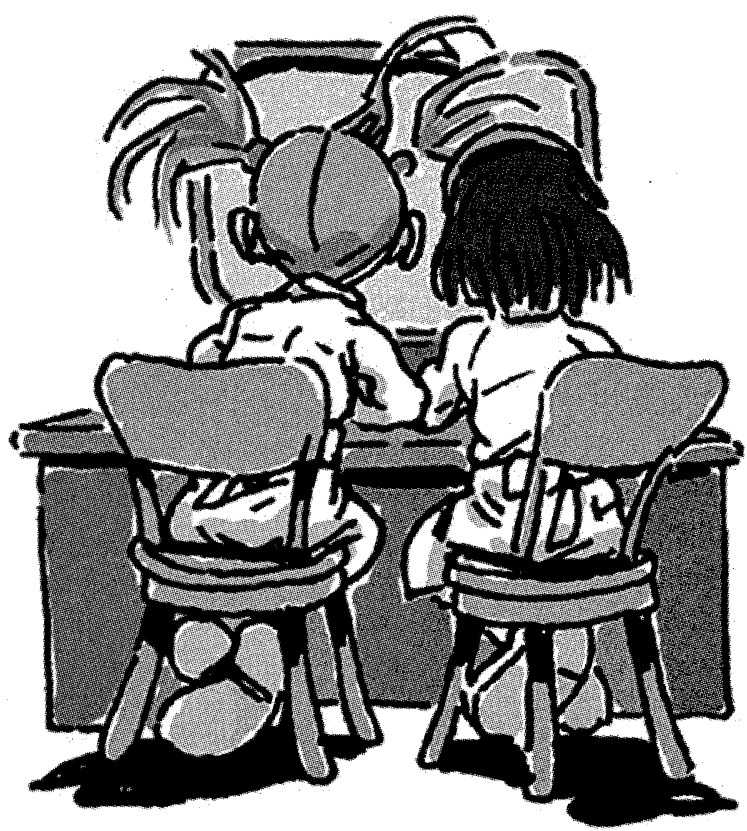

La realidad del lenguaje se está modificando vertiginosamente, sin que ni los teóricos más reconocidos ni las escuelas lingüísticas imperantes se hayan planteado la posibilidad de una nueva teoría para explicar los hechos de esta inédita realidad comunicativa, o al menos la adecuación que las teorías preexistentes requieren, una vez que con Internet se han comenzado a modificar definitivamente y de un modo asombroso tanto las relaciones intra e interhablantes como los recursos y estrategias inherentes a la producción y percepción del lenguaje.

"Si Internet es una revolución, será precisamente una revolución lingüística" (Crystal, 2002: 8). De esto podemos inferir además que, 
aparte de lo tecnológico y de lo cibernético, la emergencia de la red de redes implicará mucho más de lo que nos imaginamos para el comportamiento lingüístico humano. $\mathrm{Y}$ si recordamos que lo lingüístico es la esencia de lo social-humano, pues cómo dudar que Internet modificará todo lo que tiene que ver con nuestro comportamiento colectivo.

Descartemos de entrada la posibilidad de que los grandes teóricos del estructuralismo se hubieran detenido en el fenómeno del lenguaje virtual, porque su debilitamiento como doctrina y su dispersión universal a partir de los 90 del siglo pasado ya casi dejaba por fuera esa posibilidad.

Lo que sí resulta extraño (por no decir contraproducente) es que un teórico todavía en plena actividad intelectual como Noam Chomsky, sin duda el más relevante de la lingüística del siglo $\mathrm{xx}$, no haya considerado hasta el presente, $y$ que sepamos, el impacto que puede generar la emergencia de la red en el funcionamiento y reordenamiento de la facultad del lenguaje. Sobre todo, por las implicaciones ideológicas de este nuevo elemento tecnológico. Desde la teorización inicial planteada en Syntactic Structures (1957) hasta lo más reciente referido a los llamados postulados "minimalistas" (2002: 92-161), el autor ha pasado por alto como mínimo una referencia al asunto de cómo la red podría llegar a modificar las nociones clásicas relacionadas con el lenguaje, sus implicaciones neuronales, su desarrollo y su praxis. ${ }^{2}$

$\mathrm{Y}$ exactamente lo mismo pudiéramos argumentar en relación con los más reputados investigadores y teóricos de la llamada lingüística

2 Hay que advertir incluso que su reciente título en coautoría con Heinz Dieterich S., intitulado La aldea global, es de contenido fundamentalmente político e ideológico y nada tiene que ver con la instauración de la red. Por otra parte, el tema de la aldea global y: sus implicaciones sociológicas y comunicativas sí es discutido por Brunner (2000), y con específica alusión a Latinoamérica y el llamado "primer mundo". textual o estudios del discurso. En tanto siguen altamente interesados en las implicaciones formales, genéricas, cognitivas, políticas, sociales, raciales y laborales del discurso, y principalmente del discurso público oral e impreso en papel, muy poca preocupación han demostrado en sus últimas publicaciones por la repercusión que el fenómeno de Internet pueda tener para el desarrollo y la praxis de las habilidades y estrategias discursivas en general (van Dijk, 1997a, 1997b, 1999, 2003; Wodak y Meyer, 2003; Renkema, 1999; Fairclough, 1995, y Charaudeau, 2003).

Entre las pocas excepciones en ese sentido, destacan los estudios de David Crystal, quien ha pasado a convertirse en una de las más autorizadas voces sobre el tema, tanto en el ámbito anglófono como en el hispanohablante. Dos interesantísimos y muy profusos volúmenes ha dedicado este autor a Internet, a su juicio, la tercera y más impactante revolución ocurrida en relación con el "edificio" o "museo" del lenguaje, como él suele denominar este territorio inherente a la esencia de lo estrictamente humano (Crystal, 2002, 2005). Es precisamente él quien ha señalado que "[...] varios de los supuestos lingüísticos que dábamos por ciertos en el siglo $\mathrm{xx}$ están teniendo que ser revisados en el siglo xxI" (Crystal, 2005: 147).

Ya son innegables los efectos que Internet ejercerá tanto en los modos clásicos de lectura y escritura (Cassany, 2006) ${ }^{3}$ como en el debilitamiento del fetichismo hacia el libro impreso en papel y la emergencia de unas comunidades que pasarán a controlar la organización y difusión del conocimiento. Si bien Pierre Mounier ha catalogado Internet como "una especie de club muy ce-

\footnotetext{
3 "Para los niños de hoy, adultos del mañana, leer y escribir ya debería ser manejar webs, elaborar hipertextos, correos electrónicos, conversar a través de chat, o utilizar programas de verificación ortográfica y traducción asistida, además de tomar notas con papel y lápiz y leer libros de tapa dura" (Cassany, 2006: 184).
} 
rrado de aristócratas iguales entre sí” (2002: 47), no deja de ser cierto que su expansión y diversificación es cada vez mayor. Además, los cambios emergentes en el lenguaje de la red èstán incidiendo en alteraciones del lenguaje en general.

Ésta es una idea fundamental para la formulación de una teoría de la comunicación que cubra los nuevos espacios del lenguaje virtual y sus implicaciones para la cognición. Dichas reformulaciones deberán pasar por el hecho, resaltado por Edgar Morin (1998), según el cual la ciencia del lenguaje, la lingüística, no debe "embuclarse" sobre sí misma. Debe abrirse hacia las posibilidades que ofrecen otras ciencias, principalmente si desea cubrir todas las facetas del lenguaje y no sólo los aspectos referentes a las llamadas-lenguas naturales. Esa opción de "autoembuclarse" ha sido precisamente una de las limitantes que ha servido de eje transversal a todas las teorías precedentes, las estructuralistas, las generativistas y las discursivas. La misma teoría del texto o lingüística textual, que se autoproclama como "interdisciplinaria" o "translingüística" (Charaudeau y Maingueneau, 20024) se ha desarrollado con una muy marcada inclinación hacia lo estrictamente verbal. Y ése es un obstáculo que debe superar toda nueva formulación teórica que pretenda salir de los límites de la lingüística clásica y explicar los cambios por venir dentro de las relaciones comunicativas entre los usuarios del lenguaje.

Coincido plenamente con los planteamientos de Bastardas Boada (2003), quien postula la necesidad de integración de la lingüística con otras disciplinas, conjuntamente con la adaptación a nuevos paradigmas y metodologías menos restrictivos y más abarcadores, como, por ejemplo, el del llamado pensamiento complejo, liderado por Edgar Morin.

\section{Las comunidades lingüísticas virtuales}

Por encima de las dificultades a las que razonablemente suelen referirse los sociólogos y

\footnotetext{
4 "La linguistique textuelle, qui émerge vers la fin des années $60[\ldots]$ ne se présente pas comme une théorie de la phrase étendue au texte, mais comme une «translinguistique»" (Charaudeau y Maingueneau, 2002: 345).
}

los sociolingüistas a la hora de definirlas adecuadamente, el solo ingreso a la red mediante un buscador es señal inequívoca tanto de la existencia indudable de múltiples comunidades virtuales como de la convicción de sus miembros a la hora de reconocerse como tales. La definición más aproximada que puede hacerse de una comunidad virtual es la que la emparenta con una "multitud invisible" (Smith, 2003) cuyo muy variable y ocasional número de integrantes en la mayoría de los casos sólo se reconocen a través de sus manifestaciones comunicativas electrónicas (lingüísticas; icónicas, visuales, sonoras, etc.) y por el hecho de que comparten unos intereses y un espacio imaginario comunes. Respetando las normas inherentes al grupo, cada miembro tiene la libertad de delinear su propia identidad, simbolizada casi siempre mediante un apodo o máscara y una actitud lingüística específica, y ostenta la potestad de compartir con sus pares a través de relaciones hiperpersonales sincrónicas (caso del chateo, por ejemplo) o asincrónicas (grupos de discusión, listas de correo electrónico, mensajería telefónica) (Yus, 2001: 49-64, y Fraca, 2006). ${ }^{5}$

Aquí deseo llamar la atención sobre los cambios de algunas nociones básicas de la teoría lingüística en uso que seguramente requerirán de una reformulación futura y que, desde la reordenación de la lengua misma, tendrán una incidencia notoria en la creación y recepción lingüístico-literarias. En tal caso, habremos de pensar más bien en una teoría comunicacional mucho más expansiva que las teorías lingüís-

\footnotetext{
5 No obstante, tampoco podemos llamarnos a engaño y creer que la libertad que hoy ofrece Internet será eterna. Ya se conoce la existencia de un macroproyecto emprendido por varias universidades estadounidenses conjuntamente con el Departamento de Defensa de los Estados Unidos. Se le ha denominado Internet 2 e inicialmente tiene la apariencia de una estructura académica. Ante el temor hacia la libertad aparentemente excesiva que hasta ahora ha ofrecido el medio, uno de los objetivos principales de esta propuesta radica en la posibilidad de establecer mecanismos de censura que permitan identificar plenamente, localizar y, cuando sea el caso, penalizar a los usuarios. Detalles acerca de Internet 2 y sus implicaciones para el futuro de la red pueden obtenerse en Watson (2007). También hay interesante información sobre esto en http://es.wikipedia.org/wiki/Internet2.
} 
ticas actuales, no exclusivamente referida a las lenguas centradas en la doble articulación. Una nueva teoría lingüística que de verdad se fundamente en que el lenguaje humanò es mucho más que palabras. Desde lo más general a lo más particular, se trata de las nociones de cognición, identidad, relaciones lógicas y linealidad, espacialidad, temporalidad y velocidad. Seguramente esto traerá importantes reajustes a disciplinas como la psicolingüística y la semiótica.

\section{Los ejes conceptuales y sus reajustes}

En lo que se refiere a las habilidades cognitivas generales, habrá que reajustar la hegemonía que hasta ahora ha ejercido el hemisferio izquierdo del cerebro. Como especie, hemos entrado de ese modo en una etapa cognitiva de paralelismo cerebral bihemisférico.

Bien sabemos que el paradigma hemisférico-izquierdista ha impactado durante mucho tiempo la actividad humana en general, desde la filosofía escolar hasta la concepción ideológica de las ciencias, al considerar, por ejemplo, la matemática como centro y eje del resto del espectro científico. Y la teoría psicolingüística no ha sido la excepción en ese sentido (Barrera Linares y Fraca de Barrera, 1999: 57-68). De haberse "comportado" hasta ahora como un componente cerebral aparentemente subsidiario para efectos del lenguaje, el hemisferio derecho habrá de convertirse en el principal motor de muchas de las actividades implícitas en el manejo del universo virtual. Baste con vislumbrar nada más los efectos y consecuencias que traerá este cambio de paradigma para la ejecución de las tareas multimedia que deben ejercer los usuarios habituales de Internet.

Y será muy natural que esta revitalización del hemisferio derecho y el surgimiento de nuevas actividades requeridas por el manejo de los equipos acarree modificaciones anatómicas que nos permitan "acomodarnos" como seres sociales a la nueva situación (pensemos, por ejemplo, en fenómenos de conducta ya en pleno uso como la "pulgarización", el uso de teclados, la postura corporal frente al monitor). Como especie, hemos entrado de ese modo en una etapa cognitiva de paralelismo cerebral bihemisférico: la domi- nancia de un lado del cerebro sobre otro podría dejar de ser una premisa psicolingüística y eso incidirá notablemente en toda la cultura humana, incluyendo, por supuesto, la cultura lingüística, explicable ahora con base en unas relaciones semióticas globales, no parceladas a partir de los mecanismos de percepción y producción (sonoros, visuales, táctiles, etc.)

Además, también hay que decir que esta tecnología podría estimular la disminución o debilitamiento de algunas funciones primordialmente humanas. Pensemos, por ejemplo, en cuán importante seguirá siendo la capacidad de memorizar (datos, fechas, cifras, acontecimientos y hasta conceptos o procesos) si las máquinas se están convirtiendo en nuestras bases más confiables de datos: unas cuantas letras o números (o una combinación de ambos) son a veces el único recurso requerido para tener acceso a cualquier tipo de conocimiento que en el pasado requirió de nuestra memorización y procesamiento. Seremos así "archivadores de claves" que nos faciliten el acceso a los soportes tecnológicos y, por ende, a las fuentes de información. Con lo que a su vez se debilitará la capacidad de selección para abstraer y también habrá un decremento notorio de la estrategia de síntesis (con el consecuente incremento de la habilidad para esquematizar). Casi como vivir lingüísticamente en un universo de "palabras clave". Como señala Espen Aarseth: "Provisto de un buen buscador y una biblioteca digital, cualquier fracasado universitario puede pasar a ser un culto investigador, citando a los clásicos sin haberlos leído" (2004: 130).

De esa manera Internet nos ayuda a acumular mucha información, mas no a procesarla. $Y$ con el carácter efímero de los conocimientos de la actualidad, esa situación se confirma más aún. Aparte de lo mágico, lo mítico y lo práctico que lo caracteriza, he allí también uno de sus riesgos fundamentales.

El tópico anterior conduce además al tema de la identidad, en cuanto forma de autorrepresentación discursiva de lo que somos. Como usuarios del lenguaje asumiremos actitudes de omnisciencia y omnipotencia a la hora de manipular la "realidad", cosa que podremos hacer a nuestro antojo. Las manifestaciones discursivas a través de la red nos permiten ser alguien hoy y dejar de serlo mañana, por lo que ya algunos vis- 
lumbran la pérdida de la noción clásica de identidad a través del lenguaje. Allí podemos ser uno y muchos al mismo tiempo, con lo que obviamente se modificará también el tradicional concepto de lo ficticio. Desde la cultura escrita convencional, nos hemos empeñado en separar radicalmente la ficción de la no ficción, lo real de lo imaginario, pero también hemos padecido históricamente la necesidad de vivir lo imaginado e inmortalizarnos a través de ello. ¿No es ése el fin último de la creación literaria? Pues bien, si aceptamos que el acceso a Internet viene a romper los límites entre el mundo físico y los mundos imaginarios, pareciera ése el camino hacia un deseo ancestral de la humanidad: habitar dentro de un ámbito que podamos acomodar a nuestro antojo.

La "realidad real" es ahora otra. Y naturalmente dicha situación implicará a su vez la separación entre lenguaje y cuerpo. Asunto muy importante para una discusión en el campo de la filosofía del lenguaje.

Este mismo asunto de la identidad se relaciona con el dominio que puedan pasar a ejercer algunas lenguas sobre otras. David Crystal (2005) ha alertado ya acerca del carácter de "lengua global" que ha comenzado a adquirir el inglés como lengua mayoritaria en la red. De no atenderse este asunto con políticas oficiales inmediatas y eficaces, estaríamos amenazados por la emergencia de una hegemonía cultural y lingüística peligrosa para las comunidades que no sean anglohablantes y, por supuesto, para la preservación de la identidad colectiva que es propia de cada lengua.

Por otra parte, la cultura impresa tradicional se fundamentó de modo muy arraigado en el desarrollo del.1lamado pensamiento lógico (lineal, secuencial, organizado, jerarquizado, analítico, focalizado en los objetos, abstracto). La nueva cultura impresa virtual nos obliga a redefinir esta actitud en una apertura hacia el llamado sujeto lector multimedia o hipersensor. Podría decirse que incluso la primacía de lo narrativo para la cultura humana debería ser objeto de una nueva discusión. ${ }^{6}$

6 A fin de ampliar las posibilidades de discusión sobre lo que Internet puede significar como fenómeno de cambio, me permito referir aquí el trabajo de Sifontes Greco (2006): una monumental fuente de ideas y de muy acer-

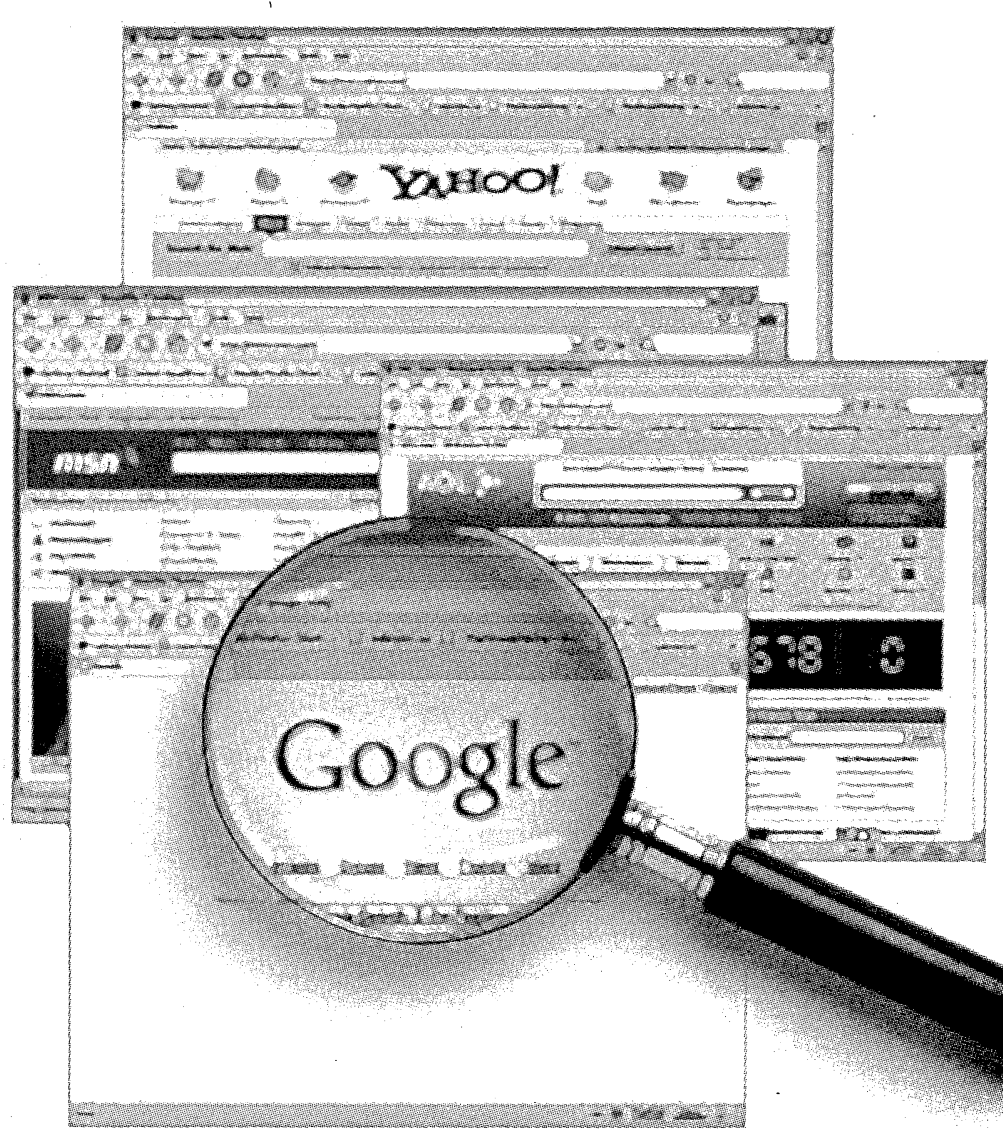

Como ya apunté arriba, en esta exigencia se potenciarán las funciones del hemisferio derecho para favorecer espacios cognitivos de pensamiento intuitivo, global, diversificado, sin orden aparente, concreto. Edgar Morin (1988) asocia estas tendencias con lo "masculino" y lo "femenino", respectivamente, lo que nos permite percibir una futura integración que rompa con dichas asociaciones culturales, instauradas ideológicamente desde su misma génesis: "Se puede pensar -afirma Morin- que la bipartición cultural masculino-femenino (a su vez consecuencia transformada y mediatizada de la bipartición biológica masculino-femenino) retroactúa desde el momento del nacimiento sobre la organización bihemisférica del cerebro y por tanto sobre el conocimiento

tadas reflexiones referentes a la virtualidad y sus implicaciones para la museística contemporánea y para (re) identificar-cuestionar el sistema mediante el cual hemos conformado tanto la narratividad y la representación del mundo como las coordenadas conceptuales para identificarlo, analizarlo y fijarlo como conocimiento. 
mismo" (101). El camino previsible es un desarrollo inevitable del llamado pensamiento complejo (Morin, 2001 y Roger Ciurana, 1997), en el cual la actividad de la escritura (y la lectura, naturalmente) será motorizada por un tejido rizomático en el que participen ambos hemisferios a fin de favorecer la multipercepción, la hiperconexión y la apreciación global de los hechos, con la consecuencia de unas relaciones de coherencia distintas a las que hasta ahora hemos manejado tanto en la lengua como en la literatura.

Adicionalmente, el ciberespacio no es el espacio convencional. Ese entorno se ha convertido en el nuevo ámbito social ilimitado, sin mediaciones temporales ni restricciones horarias; allí podemos coincidir con muchos pares sin los peligros ni las amenazas de los clásicos espacios públicos urbanos (Robins, 2004: 22). Pasaremos a ser entonces una verdadera sociedad de "amigos invisibles", una comunidad de "crisálidas" (Paul Virilio, 1993, citado en Robins, 2004): sujetos embutidos en un pequeño espacio donde sólo son necesarias las manos y el cerebro, enfrentados a una pantalla que, alimentada por un computador personal, facilita vivir en el mundo sin padecerlo). ${ }^{7}$ Nos acercaríamos a una nueva dimensión de la convivencia en grupo, el surgimiento de una nueva categoría caracterizada por la socio-individualidad: modo contemporáneo de continuar siendo individuo integrado a diversos grupos sociales sin ser parte material de ellos, habitantes de espacios de "soledad compartida", como lo denomina Fraca (2006). Mediante la virtualidad que caracteriza a Internet el ser humano puede crear y recrear los lugares a su antojo, o incluso destruirlos de acuerdo con sus requerimientos. Los espacios serán entonces lugares efímeros y cambiantes.

¿Cuál tiempo, cuál hora? Independientemente de los husos horarios, una comunidad virtual integrada por sujetos ubicados en distintos lugares del mundo-puede estar en contacto e interacción permanente, no importa dónde sea de no-

7 "[...] el pensamiento complejo integra lo más posible los modos simplificadores de pensar, pero rechaza las consecuencias rutilantes, reduccionistas, unidimensionalizantes y finalmente cegadoras de una simplificación que toma por reflejo de aquello que hubiere de real en la realidad" (Morin, 2001: 22). che, dónde sea de día, dónde esté amaneciendo, anocheciendo o atardeciendo. En ese contexto, la noción temporal (el día, la noche) se vuelve una ficción creada por el hombre, una noción que ha perdido su operatividad y.utilidad para organizar el universo, puesto que ya no son las presencias del sol y la luna los íconos orientadores del transcurso del día. Un ejemplo cotidiano de esta nueva situación es que ya a nadie le preocupa si el otro con el que requerimos comunicarnos está disponible o no para el momento en que pretendemos abrir con él o ella un procedimiento comunicativo. Supuestamente, siempre estamos a la disponibilidad del otro. Ya no tenemos ni àctividad ni tiempo "para nosotros", ni supuesto período para nuestra privacidad: el correo, la mensajería celular, el chateo, los grupos de discusión nos acosan a cualquier hora del día. Y si no que lo digan los profesores que ofrecen sus direcciones electrónicas o números de telefonía móvil a los grupos de estudiantes con los que trabajan. La ocasión para escribirles es cualquier hora a la que se les ocurra hacer cualquier tipo de pregunta, sin plantearse la posibilidad de que esté ocupado en alguna otra actividad o no. Lo que se complementa con la expectativa sobre la inmediatez con que aspira el interlocutor a que se le responda.

\section{Si recordamos que lo lingǘístico es la esencia de lo social-humano, pues cómo dudar que Internet modificará todo lo que tiene que ver con nuestro comportamiento colectivo.}

Por último me voy a referir a lo que tiene que ver con lo que ha pasado a significar la velocidad en el ámbito de Internet y sus consecuencias para la vida cotidiana.

Ya con la revolución industrial habíamos vivido un cambio en la noción de velocidad del que posiblemente ni siquiera nos dimos por enterados. Digamos que la invención del barco de vapor, del ferrocarril y del automóvil generó en la cultura humana una revolución que aceleró los tiempos de emisión y respuesta de cualquier estímulo, incluida en ello la conducta comunicativa. Un efecto similar se ha generado con la impo- 
sición de Internet en la cultura contemporánea. Lo que para una generación anterior significaba "rápido" o "veloz" ha pasado a la más lerda de las lentitudes para las nuevas generacionès. Esto se percibe tanto en la emisión de estímulos como en la capacidad de respuesta inmediata que algunos usuarios esperan de nosotros cuando manipulamos máquinas o ejercemos algún otro tipo de tarea. La cotidianidad actual está repleta de ejemplos vinculados con esta nueva diferenciación entre lo rápido y lo lento. En algunas de nuestras conductas, ya no parecemos estar al nivel de exigencia que recibimos de parte de las generaciones más jóvenes. Lo que para otros alguna vez significó "veloz" hoy puede percibirse como una capacidad retardada de respuesta ante los requerimientos de algunos de nuestros interlocutores.

Los efectos del cine, de la televisión y, principalmente, de Internet, han modificado sustancialmente las nociones de rapidez y lentitud. A simple modo de ejemplo, pensemos solamente en el lapso que se requería hace dos décadas para establecer un lazo comunicativo con alguien que está al otro lado mundo y el que se requiere ahora, o en el período que tomaba buscar una información hace veinte años en una biblioteca física cualquiera y lo que significa obtenerla hoy a través de las bibliotecas virtuales.

Y más aún, en lo que respecta a lo actitudinal, digamos que, sin importarle si está haciendo uso de un sistema virtual sincrónico (como el chateo) o asincrónico (como el correo electrónico o la mensajería telefónica celular), el emisor contemporáneo de un mensaje virtual aspira siempre a una respuesta inmediata de parte del interlocutor. Si a esto sumamos la condición de individuos multitarea que nos exige la contemporaneidad, no pasará mucho tiempo para que quienes nacimos a mediados del siglo $\mathrm{xx}$ estemos en franca situación de distonía con las generaciones emergentes.

\section{Conclusión}

Si a partir de Internet están cambiando las múltiples variables relacionadas con la comunicación humana, es obvio que también ha- brán de modificarse los soportes y códigos que le sirven de vehículo. $Y$ ante las exigencias manipulatorias y neuronales de tales soportes y códigos, seguramente vendrán reajustes diversos relacionados con la cognición y con la conceptualización. Estamos viviendo la etapa transicional de una cultura lingüística a otra y eso deberá traer una nueva formulación teórica que dé cuenta de las implicaciones psicolingüísticas de tal fenómeno y de sus repercusiones en la actividad comunicativa humana. Un reto importante no sólo para la teoría lingüística sino también para las instituciones escolares y para la sociedad en general.

Igual que está ocurriendo en la ciberlingua (como la denomina Fraca, 2006), la ciberliteratura correrá también con las consecuencias de las reformulaciones a que están sujetos todos los conceptos generados a partir de la teoría lingüística ortodoxa. Identidad autoral, relaciones lógicas, linealidad y secuencialidad, concepción del tiempo y del espacio, velocidad de comunicación y procesamiento psicolingüístico precisan de una nueva teoría lingüística que les dé sentido ante la realidad comunicacional emergente, realidad que no sólo afectará a los usuarios directos de la red, sino que seguramente modificará a todo el "edificio" del lenguaje: dentro y fuera de Internet.

En Internet se ha modificado sustancialmente la dicotomía falsedad/realidad. Todo puede ser real y todo puede ser ficción, sin que haya manera de comprobar lo contrario. El lector puede asumir una posición determinada mas no verificarla ni comprobarla: la lengua de la red no es entonces tan "verdadera o falsa" como la ortodoxa lengua cotidiana y este principio es fundamental tanto para la lengua en general como para la ciberliteratura, donde además podrían perder sentido conceptos de la literatura tradicional como "plagio", "originalidad", "documentación fidedigna”, "autoría concreta”, etc. Ó

\section{Bibliografía}

Aarseth, E. (2004), "La literatura ergódica", en Sánchez-Mesa, D. (comp.), Literatura y cibercultura, Madrid, Arco Libros, pp. 117-146. 
Barrera Linares, L. (2005), La lengua y la literatura en-Red-a-das: nuevos temores hacia antiguas estrategias comunicativas . [discurso de incorporación como Individuo de Número a la Academia Venezolana de la Lengua], Caracas, AvL.

- y Fraca de Barrera, L. (1999), Psicolingüistica y desarrollo del español II, Caracas, Monte Ávila Editores Latinoamericana.

Bastardas Boada, A. (2003), "Lingüística general: elementos para un paradigma integrador desde la perspectiva de la complejidad", en LINRED [disponible en www.uah.es/linred, consulta 5 de mayo de 2006].

Bohn, D. (1980), La totalidad y el orden implicado, Barcelona, KAIROs.

Brunner, J. J. (2000), "Cibercultura: la aldea global dividida", mesa redonda sobre cibercultura, Hannover [en línea], disponible en: http://mt.educarchile.cl/mt/jjbrunner/archives/2005/08/cibercultura_la.html.

Cassany, D. (2006), Tras las lineas. Sobre la lectura contemporánea, Barcelona, Anagrama.

Castells. M. (2000), La galaxia Internet. Reflexiones sobre Internet, empresa y sociedad, Madrid, Areté.

Charaudeau, P. (2003), El discurso de la información, Barcelona. Gedisa.

- y Maingueneau, D. (2002), Dictionnaire D'analyse du Discours, París, Seuil.

Chomsky, N. (2002), On Nature and Language, Cambridge, cup.

-y Dieterich S., H. (1997), La aldea global, Navarra, Txalaparta.

Crystal, D. (2002), El lenguaje e Internet, Madrid, Cambridge University Press.

- (2005), La revolución del lenguaje, Madrid, Alianza.

Fairclough, N. (1995), Critical Discourse Analysis, Londres, Longman.

Fraca de Barrera, L. (2006), La ciberlingua. Una variedad compleja de lengua en Internet, $\mathrm{Ca}-$ racas, Instituto de Investigaciones Lingüísticas y Literarias Andrés Bello, upeL.
McLuhan, M. (1969), La galaxia Gutenberg. Génesis del homo typographicus, Madrid, Aguilar.

Morin, E. (1988), El método. III. El conocimiento del conocimiento.Libro primero. Antropología del conocimiento, Madrid, Cátedra.

- (1998), El método. Las ideas, Madrid, Cátedra.

- (2001), Introducción al pensamiento complejo, Barcelona, Gedisa.

Mounier, P. (2002), Los Dueños de la Red, Madrid, Popular.

Renkema, J. (1999), Introducción a los estudios sobre el discurso, Barcelona, Gedisa.

Robins, K. (2004), "El ciberespacio y el mundo en que vivimos", en Sánchéz-Mesa, D. (comp.), Literatura y Cibercultura, Madrid, Arco Libros, pp. 199-232.

Sifontes Greco, L. (2006), Literatura sobre 10 virtual. Espacios y lenguaje de una narrativa contemporánea [inédito: trabajo de ascenso para titular], Caracas, Universidad Simón Bolívar.

Smith, M. (2003), "La multitud invisible en el ciberespacio", en Smith, M. y Kollock, P. (edits.), Comunidades en el ciberespacio, Barcelona, uoc, pp. 227-257.

van Dijk, T. (1999), Ideología, Barcelona, Gedisa.

- (2003), Dominación étnica y racismo discursivo en España y América Latina, Barcelona, Gedisa.

- (edit.), (1997a), Discourse as Structure and Process, Londres; sage Publications.

- (edit., 1997b), Discourse as Social Interaction, Londres, sage Publications.

Watson, S. (2007), "Científicos usamericanos quieren desembarazarse de la red de Internet" (trad. Manuel Talens) [en línea], disponible en: http://www.rebelion.org/noticia. php?id=49932, consulta: 27-04-2007.

Wodak, R. y Meyer, M. (2003), Métodos de análisis critico del discurso, Barcelona. Gedisa.

Yus, F. (2001), Ciberpragmática. El uso del lenguaje en Internet, Barcelona, Ariel. 